\title{
An Intelligent Cloud-based Health Care Architecture for Long-term Care
}

\author{
Zheng-Yang Lin, I-Ching Hsu ${ }^{*}$ \\ Department of Computer Science and Information Engineering, \\ National Formosa University, Taiwan \\ *Corresponding Author: hsuic@nfu.edu.tw
}

\begin{abstract}
An aging society is a common trend in all countries. Long-term care is a policy that must be implemented in an aging society. Therefore, how to develop an intelligent cloud-based information system to efficiently support long-term care and manage related resources is an important issue. In recent years, open data, artificial intelligence and cloud computing are popular information technologies. This study proposes an Intelligent Cloud-based Health Care Architecture (ICHCA) that is composed of data presentation module, data source module, intelligent module and cloud computing module to facilitate the development of long-term care information systems. This study develops a Cloud Medical Knowledge Platform(CMKP) prototype to verify the feasibility of the proposed ICHCA.
\end{abstract}

Keywords: Long-term Care, Ontology, Cloud Computing.

\section{Introduction}

The aging society is a common social phenomenon in all countries. Long-term care is mainly for those who need care service provided by others due to the disability in everyday life. These cases are mostly the elderly aged above 65. Taiwan government proposed a ten years long-term care 2.0 project to support aging society. The lack of long-term care resources is an issue that needs urgent improvement. Therefore, how to use information technologies to make good use of long-term care resources is a topic worth studying. This study proposes an Intelligent Cloud-based Health Care Architecture (ICHCA) that is composed of data presentation module, data source module, intelligent module and cloud computing module to facilitate the development of long-term care information systems.

The proposed ICHCA adopts Association Rule,
Semantic Web[1], Open Data[2] and Cloud Computing[3] technologies to facilitate providing correct and appropriate long-term care services to promote development of long-term care information system. The Semantic Web is an extension of the traditional web, which provides RDF-based metadata to facilitate developing an intelligent application. Many studies adopt Semantic Web to build intelligent applications in various domains. Cloud computing has become one of the most promising information solutions and business trends in recent years. Many cloud-based information systems have enabled easy access to the cloud-based resources that they provide. Anyone can create a new integrated application with these cloud-based resources.

\section{Related Work}

In recent years, several research studies [4-6] have focused on various information technologies, including cloud computing, Internet of Thing[7], Semantic Web, etc., to enhance health care information system. Hadoop [8] is a popular cloud computing platform that is capable of effectively processing and storing big data, which provides distributed computing architecture. It mainly uses Hadoop Common to provide the library needed by other modules. The core is composed of HDFS and Map Reduce. The YARN cluster manager is imported into Hadoop2 version [9].

Spark [10] is an open-source platform for fast cluster computing. The major characteristic is that it uses In-memory technique, and finally stores the computing results in the hard disk, so as to reduce the I/O time in the hard disk. Spark architecture consists of the cluster manager, core and internal library. The Spark Core includes the basic functions such as scheduling and memory management, as well as the API of the major Resilient Distributed 
Datasets(RDD). The cluster manager is a Standalone Scheduler built in Spark. The internal library provides Spark SQL, Spark Streaming, MLlib and GraphX. The distributed resource manager Apache Mesos is a distributed system core. Mesos considers the multi-node resources as a single high-performance computer, including the CPU, memory, hard disk and other computing resources on the node. It shares the available resources on the node among different applications without mutual interference.

\section{Intelligent Cloud-based Health Care Architecture}

The section describes an Intelligent Cloud-based Health Care Architecture (ICHCA). As shown in Fig. 1, ICHCA is a generalized and conceptual framework which is composed of 4 modules: data presentation module, data source module, intelligent module and cloud computing module, which will be respectively introduced as below.

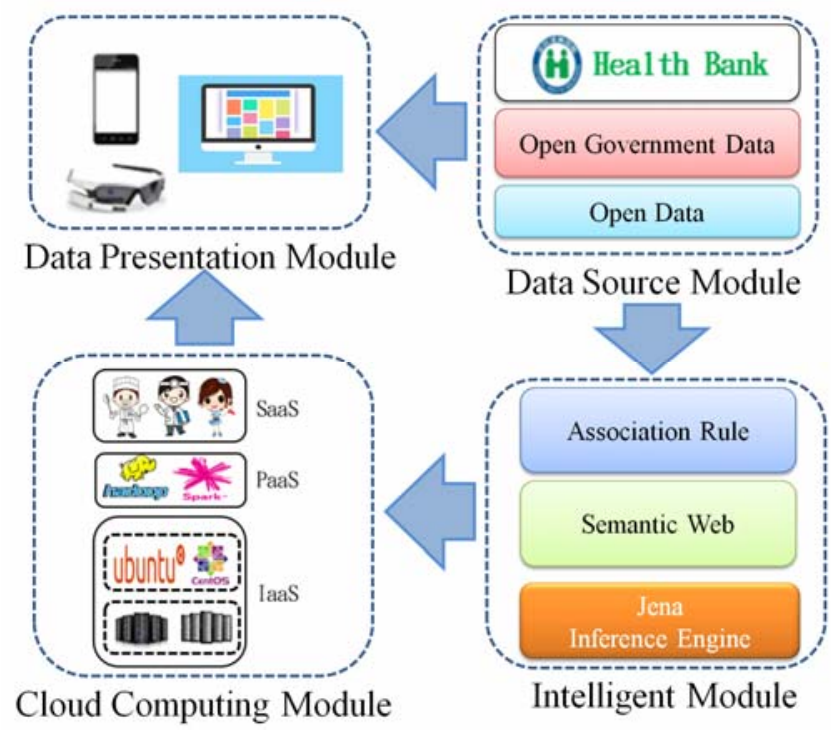

Fig. 1 Intelligent Cloud-based Health Care Architecture

\section{Data Presentation Module}

Data Presentation Module contains various client devices, for example, mobile phone, wearable device, desktop computer, etc, to provide data display for users. Data display supports HTML-based or App-based presentation via Internet.

\section{Data Source Module}

Data Source Module contains open resource and Health Bank. Open resource is a kind of open data, called Open Government Data (OGD) [11] that is mainly provided by government. The Health Bank is e-health information system developed by Taiwan government to provide personal health information. Taiwanese citizens can get their own medical information through the Health Bank. Open data is a global trend for data sharing. This module integrates the open datasets in the long-term care field, such as the geographic map of long-term care.

\section{Intelligent Module}

Intelligent Module integrates Association Rule with Semantic Web into Cloud Computing environment. Association Rule extracts the relationship between different datasets to capture implicit rules. These implicit rules can be used on top of ontology. The ontology is a mainly technology of Semantic Web that is a Web-based knowledge presentation in the internet. Jena [12] is an inference engine developed by Apache to provide an ontology-based reasoner for semantic-based markup language, including RDF[13], RDFS[14], and OWL[15].

\section{Cloud Computing Module}

Cloud Computing Module consists of Infrastructure as a Service (IaaS), Platform as a Service (PaaS) and Software as a Service (SaaS). SaaS provides services to end users, while IaaS and PaaS provide services to cloud application developers. The SaaS supports to develop health care information system. The PaaS is developed based on Hadoop and Spark cloud computing environment. The IaaS adopts ubuntu operation system in high-performance cluster computers.

\section{Cloud Medical Knowledge Platform}

To validate the feasibility of ICHCA architecture proposed in this paper, this study develops the Cloud Medical Knowledge Platform(CMKP) prototype based on ICHCA, as shown in Fig. 2. The illustration below is mainly based on dataflow stream.

1. Developer

1.1 Build the Ubuntu operation system, install Hadoop and Spark on the system, and then build the Association Rule and Semantic Web of Intelligent Module on Spark to make computing.

1.2 Store the computing result in CMKP.

1.3 Introduce the air quality index Open Data through API as the basis for making recommendation.

1.4 With 100 copies questionnaires of common disease and cancer reading association in txt format, find out the rules of association between diseases through Association Rule. 
1.5 Convert these rules into Rule, and use the Semantic Web technology built on cloud computing to get new inference results for OWL, RDF and Rule through Jena inference engine and store these recommendation results in CMKP following step 1.2.

2. Users and hospitals

2.1 Users and hospital practitioner can use mobile devices and desktop computers to access CMKP. For example, hospital practitioners can create new patient education documents; users can download the patient education documents.
3. Hospital

3.1 EPUB(Electronic Publication)[16] is an international standard format of electronic books proposed by International Digital Publishing Forum(IDPF). Hospital practitioners can build related medical EPUB for patient education through CMKP.

4. User

Users can access the health record in health bank and import personal health record into CMKP.

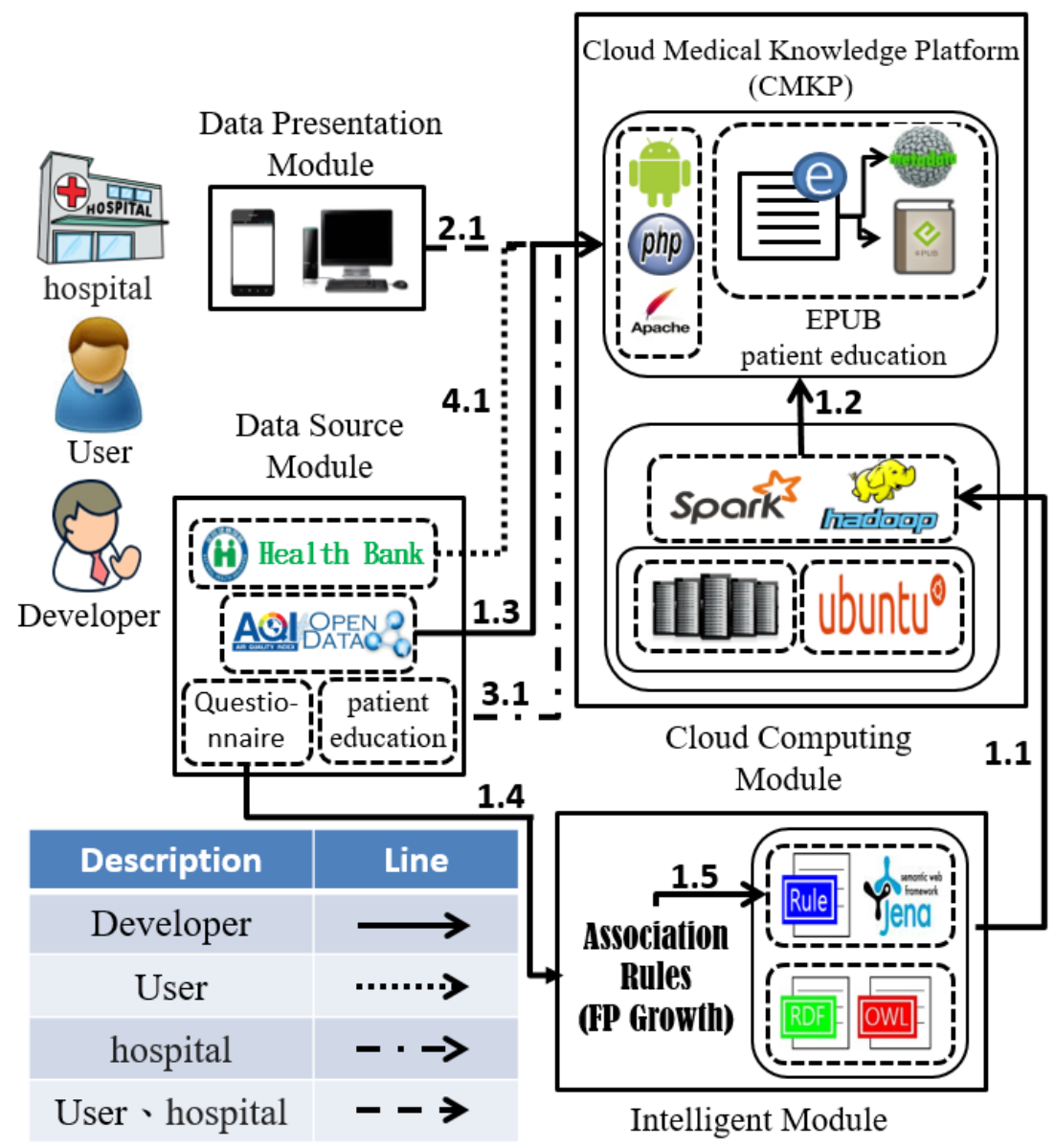

Fig 2. The dataflow-oriented CMKP based on ICHCA

\section{Ontology Development}

The Diseases Ontology of CMKP is developed based on ICD-10-CM(International Classification of Diseases, 10th Revision, Clinical Modification)[17], which codify and categorize each disease. ICD-10-CM provides over 68,000 disease codes. Some parts are selected as research example. The Diseases Ontology is coded in OWL. The UML diagram of Diseases
Ontology is shown in Fig. 3. Figure 4 shows partial OWL-based code of Diseases Ontology.

\section{Health Bank}

Health Bank is a health care information system developed by Taiwan to provide personal disease record. The CMKP converts personal disease record to an RDF-based format to invoke jena inference engine. Figure 5 shows partial code of RDF-based personal disease record. 


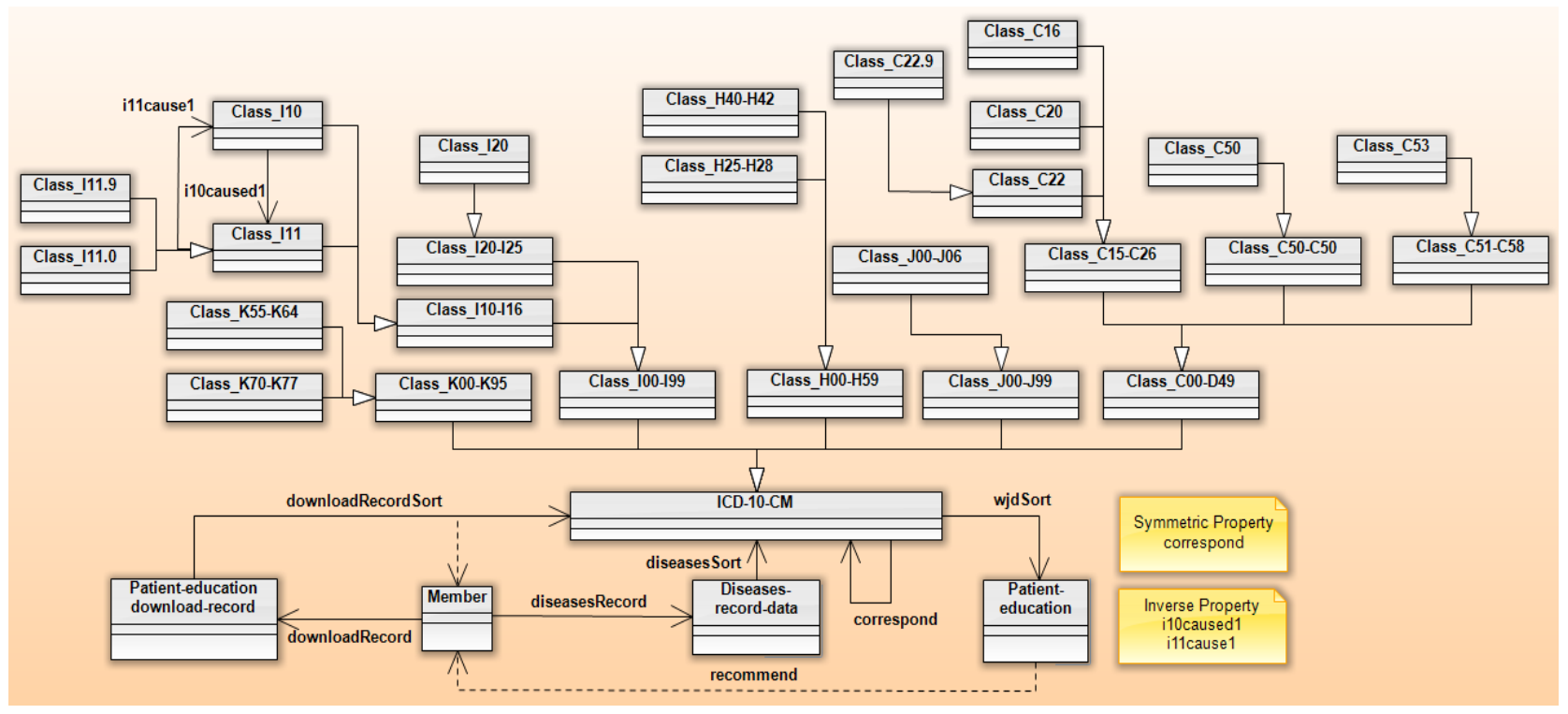

Fig. 3 UML diagram of Diseases Ontology

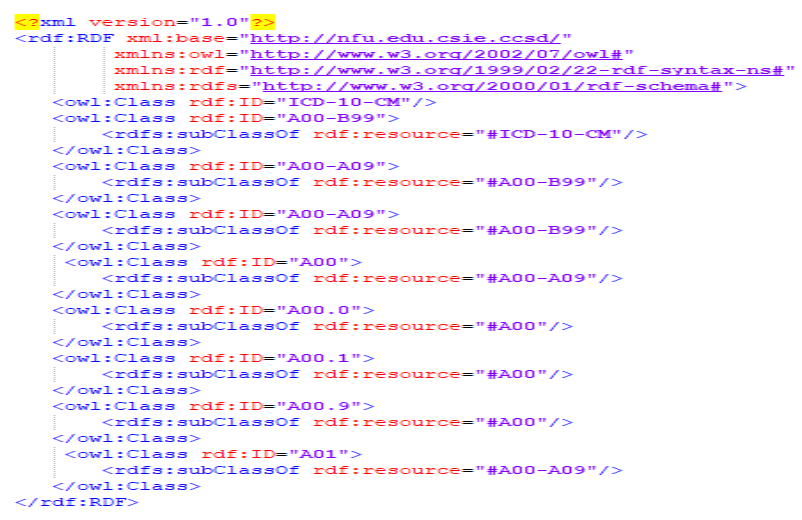

Fig. 4 Partial OWL-based code of Diseases Ontology

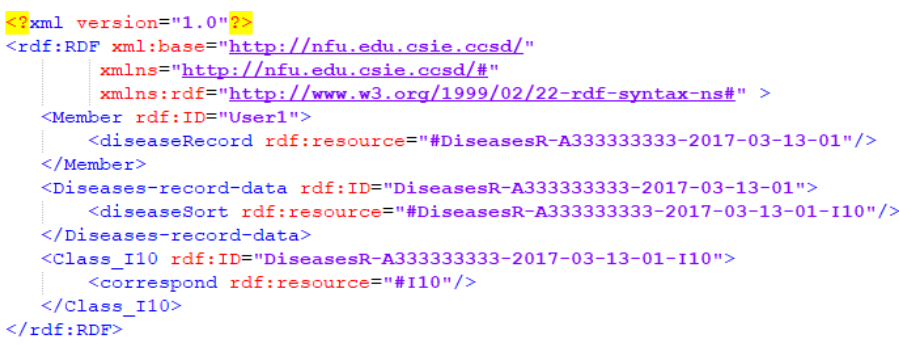

Fig. 5 Partial RDF-based code of disease record

\section{Association Rule Development}

There are 14 association rules defined on top of the Diseases Ontology. Each association rule is coded in jena-based rule. If a person subscribes a certain EPub that is published by a certain hospital, then the person is a patient of the hospital.

(?user pre:sub ?EPub)(?EPub pre:pub ?hospital)->

(?user pre:patient ?hospital)

\section{Experimental Results}

After describing the framework for enhancing the intelligent capabilities of CMKP, a preliminary experiment is performed to evaluate system efficiency based on cloud computing environment. The hadoop-based Spark cloud computing environment is composed of a single master server and six salve servers. Each server consists of $\mathrm{CPU}$ I7-2600 3.4GHz, RAM 16G, and two hard disks with capacities of $2 \mathrm{~T}$ and $500 \mathrm{G}$, respectively. This study executes two cloud computing approaches, Spark MLlib 2.1.0 (called MLlib for short) and Apache Mahout 0.8 (called Mahout for short).

This experimentation measures the data size from $100 \mathrm{M}$ to $1000 \mathrm{M}$, with $100 \mathrm{M}$ per gap. Figure 6 presents the performance of the MLlib and Mahout approaches. There are two lines: one is the execution time of Mahout for each point from $100 \mathrm{M}$ to $1000 \mathrm{M}$. It takes 74 seconds to execute until $100 \mathrm{M}$ and 179 seconds to execute until $1000 \mathrm{M}$; the other line is the execution time of MLlib. It takes 21 seconds to execute until $100 \mathrm{M}$ and 27 seconds to execute until 1000M. This experimentation showed that compared with MLlib, the execution time of Mahout is more seriously affected by the change of data set, while the performance of MLlib is better than Mahout. 


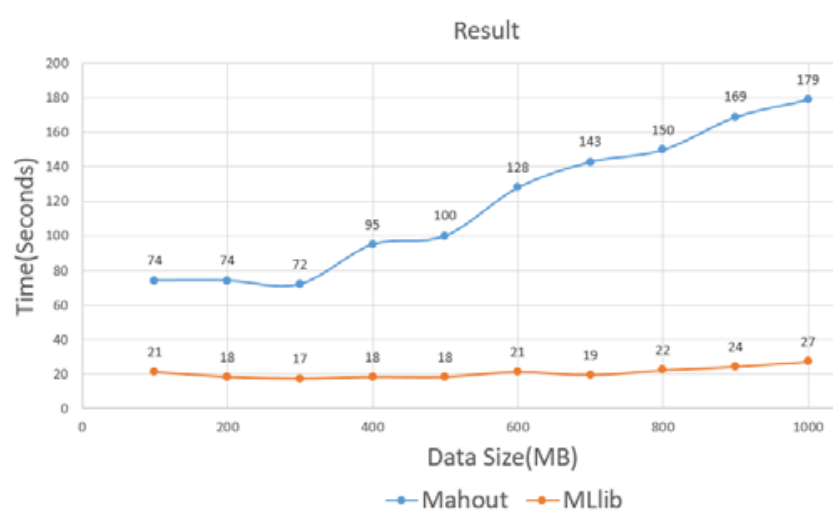

Fig. 6 Performance comparison between MLlib and Mahout

\section{Conclusions}

This study presents an Intelligent Cloud-based Health Care Architecture (ICHCA) for modular design, which consists of data presentation module, data source module, intelligent module and cloud computing module to facilitate the development of long-term care information systems. This ICHCA enables long-term care information systems to support an intelligent cloud-based health care. The Cloud Medical Knowledge Platform(CMKP) prototype is developed to verify the feasibility of the proposed ICHCA. The CMKP is evaluated using two different cloud computing approaches, Spark MLlib 2.1.0 and Apache Mahout 0.8. The experimental results show that the performance of Spark MLlib 2.1.0 is better than Apache Mahout 0.8 .

\section{Acknowledgment}

The author would like to thank the Ministry of Science and Technology, R.O.C, for financially supporting this research under Contract No. MOST 107-2637-E-150-009-.

\section{References}

1. Yu, L., A Developer's Guide to the Semantic Web2010: Springer.

2. Berners-Lee, T. The year open data went world wide. $2010 \quad$ Available from: http://www.ted.com/talks/tim_berners lee the yea r open data went worldwide.html. Access date: Aug. 21, 2018.

3. Durao, F., et al., A systematic review on cloud computing. Journal of Supercomputing, 2014. 68(3): p. 1321-1346.
4. Meng, X.J., H.Q. Cui, and R. Hua, An IoT-based remote health monitoring and management system Applied Mechanics and Materials, 2014. 571-572: p. 1176-1179.

5. Ahire, S.B. and H.K. Khanuja, A personalized framework for health care recommendation in Proceedings - 1st International Conference on Computing, Communication, Control and Automation, ICCUBEA2015: Pune, India. p. 442-445.

6. Vallerand, J., J. Lapalme, and A. Moïse, The design of an m-Health monitoring system based on a cloud computing platform Enterprise Information Systems, 2017. 11(1): p. 17-36.

7. Ammar, M., G. Russello, and B. Crispo, Internet of Things: A survey on the security of IoT frameworks. Journal of Information Security and Applications 2018. 38: p. 8-27.

8. Jam, M.R., et al., Survey on improved Autoscaling in Hadoop into cloud environments, in 2013 5th Conference on Information and Knowledge Technology2013, IEEE Computer Society Shiraz, Iran. p. 19-23.

9. Huang, W., et al., In-Memory Parallel Processing of Massive Remotely Sensed Data Using an Apache Spark on Hadoop YARN Model. IEEE Journal of Selected Topics in Applied Earth Observations and Remote Sensing, 2017. 10(1): p. 3-9.

10. Mavridis, I. and H. Karatza, Performance evaluation of cloud-based log file analysis with Apache Hadoop and Apache Spark. The Journal of Systems and Software, 2017. 125: p. 133-151.

11. Magalhães, G. and C. Roseira, Exploring the barriers in the commercial use of open government data, in 9th International Conference on Theory and Practice of Electronic Governance, ICEGOV 20162016, Association for Computing Machinery: Montevideo, Uruguay p. 211-214

12. Jena. Jena - A Semantic Web Framework for Java 2007 Available from: http://jena.sourceforge.net/. Access date: Aug. 21, 2018.

13. W3C. Resource Description Framework (RDF). 2014 Available from: https://www.w3.org/RDF/. Access date: Aug. 21, 2018.

14. Brickley, D. and R.V. Guha. RDF Vocabulary Description Language 1.0: RDF Schema. 2004 Available from: http://www.w3.org/TR/rdf-schema. 
Access date: Aug. 21, 2018.

15. Smith, M.K., C. Welty, and D.L. McGuinness.

OWL Web Ontology Language Guide. 2004

Available from: http://www.w3.org/TR/owl-guide/.

Access date: Aug. 21, 2018.

16. Conboy, G., et al. EPUB 3 Overview. 2011 Available from:

http://idpf.org/epub/30/spec/epub30-overview.html. Access date: Aug. 21, 2018.

17. Organization, W.H. International Classification of Diseases (ICD). Available from: http://www.who.int/classifications/icd/en/. Access date: Aug. 21, 2018. 